\section{Antidepressants and spontaneous abortion}

On the cover of the July 13, 2010 issue of $C M A J$ was the heading, "Use of antidepressants in pregnancy increases the risk of spontaneous abortion." The findings in the related research article by Nakhai-Pour and colleagues are based solely on prescription drugs. ${ }^{1}$ No estimate was done of actual compliance with the medications. If a woman was aware that she was pregnant, the likelihood of her taking the medication might be lower. Patients sometimes fill a prescription but then decide against taking it.

Medical contact for antidepressant therapy might be associated with pregnancy testing, making women more aware that vaginal bleeding might be a sign of spontaneous abortion.

What can be said is that filling a prescription for an antidepressant is associated with an increase in clinically detected spontaneous abortions.

\section{Don Lint MD}

Brandon Regional Health Authority, Brandon, Man.

\section{Reference}

1. Nakhai-Pour HR, Broy P, Bérard A. Use of antidepressants during pregnancy and the risk of spontaneous abortion. CMAJ 2010;182:1031-7.

CMAJ 2011. DOI:10.1503/cmaj.111-2056

We thank Lint for his comment on our article. ${ }^{1}$ Indeed, our 68\% increase in the risk of spontaneous abortion with gestational use of antidepressants was based on prescriptions filled and not actual medication intake. The potential limitations associated with this were clearly stated in our article. We also highlighted that there is enough evidence-based research showing that the majority of pregnant women having a prescription filled will take at least one dose. Hence, our main definition of exposure categorized women dichotomously as having taken at least one dose. Our main finding replicates the results of a study by Einarson and colleagues, ${ }^{2}$ who showed that, based on women's reports of actual medication intake, antidepressant use during gestation increased the risk of spontaneous abortion by $68 \%$.

In this instance, actual antidepressant intake and antidepressant use defined by prescriptions filled gave concordant findings. The novelty of our study was its large sample size, which also enabled the study of antidepressant types and dosages.

Finally, there is no evidence showing that medical contact for antidepressant therapy increases the rate of pregnancy detection.

\section{Anick Bérard PhD}

Professor, Faculty of Pharmacy, University of Montréal; Director, Research Unit on

Medications and Pregnancy, CHU Ste-Justine, Montréal, Que.

\section{References}

1. Nakhai-Pour HR, Broy P, Bérard A. Use of antidepressants during pregnancy and the risk of spontaneous abortion. CMAJ 2010;182:1031-7.

2. Einarson A, Choi J, Einarson TR, et al. Rates of spontaneous abortion and therapeutic abortions following use of antidepressants in pregnancy: results from a large prospective database. J Obstet Gynaecol Can 2009;31:452-6.

CMAJ 2011. DOI:10.1503/cmaj.111-2057

\section{"Hari": not embedded needles}

Park and Shim described a female patient who had countless acupuncture needles left in her body, especially around paraspinal muscles. ${ }^{1}$ They stated that "this subtype of acupuncture known as Hari involves the permanent placement of fine needles into the subcutaneous tissue," but this is not correct.

The Japanese word Hari means sewing needle, acupuncture therapy or acupuncture needle. When we use this word to mean acupuncture therapy, it means acupuncture in general. Referring to a paper by Vassiou and colleagues, ${ }^{2}$ Park and Shim explained that the retained needles are typically $1 \mathrm{~mm}$ in diameter, but this is also incorrect. The diameter of retained needles is the same as that of acupuncture needles usually used in Japan: between 0.14 and $0.20 \mathrm{~mm}$.

In Japan, we usually use the word Maibotsu-Shin for embedding acupuncture needles, but this practice is not performed anymore because it has caused many adverse events. ${ }^{3}$

As with the evaluation of efficacy in evidence-based medicine, the safety of acupuncture should be assessed through prospective surveys or controlled studies that show incidence, degree of severity and significance of differences wih control groups. Recent large-scale prospective surveys in Germany involving about 200000 patients show that serious adverse events of acupuncture are very uncommon, ${ }^{4,5}$ but some do occur. We continue to provide all those who perform acupuncture with updated information for safer practice.

\section{Hitoshi Yamashita LicAc PhD}

Professor and chair, Morinomiya University of Medical Sciences, Osaka, Japan

\section{References}

1. Park SM, Shim WJ. A hedgehog-like appearance resulting from Hari acupuncture. CMAJ 2011; June 27 [Epub ahead of print].

2. Vassiou K, Kelekis NL, Fezoulidis IV. Multiple retained acupuncture needle fragments. Eur Radiol 2003;13:1188-9

3. Yamashita H, Tsukayama H, White AR, et al. Systematic review of adverse events following acupuncture: the Japanese literature. Complement Ther Med 2001;9:98-104

4. Witt CM, Pach D, Brinkhaus B, et al. Safety of acupuncture: results of a prospective observational study with 229,230 patients and introduction of a medical information and consent form. Forsch Kimplementmed 2009;16:91-7.

5. Endres HG, Molsberger A, Lungenhausen M, et al. An internal standard for verifying the accuracy of serious adverse event reporting: the example of an acupuncture study of 190,924 patients. Eur J Med Res 2004;9:545-51.

6. Yamashita H, Tsukayama H. Safety of acupuncture practice in Japan: patient reactions, therapist negligence and error reduction strategies. Evid Based Complement Alternat Med 2008;5:391-8.

CMAJ 2011. DOI:10.1503/cmaj.111-2059

\section{Routine peripubertal circumcision?}

MacDonald deserves credit for shifting the debate on circumcision away from inconclusive quibbles about the balance of risks and benefits, ${ }^{1}$ but it is not obvious that peripubertal boys are any more capable of giving consent than a baby. They lack maturity and sufficient knowledge; boys at 11 or 12 years of age are not considered competent to consent to sexual relations with others, 